\title{
Detection of a high-mass prestellar core candidate in W43-MM1
}

\author{
T. Nony ${ }^{1}$, F. Louvet ${ }^{2}$, F. Motte ${ }^{1,3}$, J. Molet ${ }^{4}$, K. Marsh ${ }^{5}$, E. Chapillon ${ }^{6,4}$, A. Gusdorf ${ }^{7}$, N. Brouillet ${ }^{4}$, S. Bontemps ${ }^{4}$, \\ T. Csengeri ${ }^{8}$, D. Despois ${ }^{4}$, Q. Nguyen Luong 9 , A. Duarte-Cabral ${ }^{5}$, and A. Maury ${ }^{3}$
}

${ }^{1}$ Univ. Grenoble Alpes, CNRS, IPAG, 38000 Grenoble, France e-mail: thomas .nony@univ-grenoble-alpes.fr

2 Departmento de Astronomia de Chile, Universidad de Chile, Santiago, Chile

3 AIM, CEA, CNRS, Université Paris-Saclay, Université Paris Diderot, Sorbonne Paris Cité, 91191 Gif-sur-Yvette, France

${ }^{4}$ OASU/LAB, Univ. de Bordeaux - CNRS/INSU, 36615 Pessac, France

5 School of Physics and Astronomy, Cardiff University, Queens Buildings, The Parade, Cardiff CF24 3AA, UK

6 Institut de RadioAstronomie Millimétrique (IRAM), Grenoble, France

7 LERMA, CNRS, Observatoire de Paris, École Normale Supérieure, 24 rue Lhomond, 75231 Paris Cedex 05, France

8 Max-Planck-Institut für Radioastronomie, Auf dem Hügel 69, 53121 Bonn, Germany

9 Canadian Institute for Theoretical Astrophysics, University of Toronto, 60 St. George Street, Toronto, ON M5S 3H8, Canada

Received 16 July 2018 / Accepted 27 September 2018

\section{ABSTRACT}

\begin{abstract}
Aims. To constrain the physical processes that lead to the birth of high-mass stars it is mandatory to study the very first stages of their formation. We search for high-mass analogs of low-mass prestellar cores in W43-MM1.

Methods. We conducted a $1.3 \mathrm{~mm}$ ALMA mosaic of the complete W43-MM1 cloud, which has revealed numerous cores with $\sim 2000$ au FWHM sizes. We investigated the nature of cores located at the tip of the main filament, where the clustering is minimum. We used the continuum emission to measure the core masses and the ${ }^{13} \mathrm{CS}(5-4)$ line emission to estimate their turbulence level. We also investigated the prestellar or protostellar nature of these cores by searching for outflow signatures traced by $\mathrm{CO}(2-1)$ and $\mathrm{SiO}(5-4)$ line emission, and for molecular complexity typical of embedded hot cores.

Results. Two high-mass cores of $\sim 1300$ au diameter and $\sim 60 M_{\odot}$ mass are observed to be turbulent but gravitationally bound. One drives outflows and is associated with a hot core. The other core, W43-MM1\#6, does not yet reveal any star formation activity and thus is an excellent high-mass prestellar core candidate.
\end{abstract}

Key words. stars: formation - stars: protostars - stars: massive - submillimeter: ISM - ISM: clouds

\section{Introduction}

Despite the large efforts made in the past ten years to improve our understanding of the formation of high-mass stars, two competing families of models remain. In the "core-fed" or "coreaccretion" models, a high-mass star forms through the monolithic collapse of a massive, turbulent prestellar core that formed quasi-statically (e.g., McKee \& Tan 2003). This preassembled core is in virial equilibrium, similar to low-mass prestellar cores, but supported by some magnetic and/or supersonic turbulent pressure that prevents the subfragmentation of this core. A few high-mass prestellar core candidates have been reported (Bontemps et al. 2010; Duarte-Cabral et al. 2013; Wang et al. 2014) but more examples are needed to support this scenario.

On the other hand, the "clump-fed" models involve the gas mass reservoir surrounding individual cores through dynamical processes in their parental cloud. These models propose the rapid growth of cores via competitive accretion of the common cloud mass reservoir (e.g., Bonnell \& Bate 2006) or via accretion streams associated with the global hierarchical collapse of clouds (e.g., Smith et al. 2009; Vázquez-Semadeni et al. 2017). Observationally, there is a growing body of evidences in favor of dynamical, clump-fed models (e.g., Schneider et al. 2010; Csengeri et al. 2011; Peretto et al. 2013; Henshaw et al. 2014;
Louvet et al. 2016). In the empirical evolutionary sequence recently proposed for the formation of high-mass stars, the highmass prestellar core phase does not even exist and high-mass protostellar cores form from low-mass protostellar cores, which accrete further material from their parental massive dense core (MDC; Motte et al. 2018a).

Imaging cold, high-mass star-forming clouds is needed to reach a consensus on the existence of a high-mass prestellar core phase. W43, located at $5.5 \mathrm{kpc}$ from the Sun (Zhang et al. 2014), contains two of the largest groups of molecular clouds in the first Galactic quadrant, among them W43-MM1 (Nguyen Luong et al. 2011, 2013). This $6 \mathrm{pc}^{2}$ ridge has a $2 \times$ $10^{4} M_{\odot}$ mass and qualifies as "mini-starburst" because its star formation activity is reminiscent of that of starburst galaxies (SFR $6000 M_{\odot} \mathrm{Myr}^{-1}$; Motte et al. 2003; Louvet et al. 2014). Imaged with ALMA, W43-MM1 revealed one of the youngest and richest clusters of high-mass cores in the Milky Way (Motte et al. 2018b).

In the present paper, we characterize two high-mass cores located in the least clustered part of W43-MM1 and report on the possible discovery of one high-mass prestellar core. From the observations presented in Sect. 2, we derive the core characteristics using dust continuum (see Sect. 3.1) and investigate their gravitational boundedness and evolutionary status with 
molecular line observations (see Sects. 3.2 and 3.3). Core properties are then used in Sect. 4 to discuss their prestellar versus protostellar nature and the most probable physical process forming high-mass stars in W43-MM1.

\section{Observations and data reduction}

Observations were carried out in Cycle 2 between July 2014 and June 2015 (project \#2013.1.01365.S), with ALMA $12 \mathrm{~m}$ and $7 \mathrm{~m}$ (ACA) arrays and baselines ranging from $7.6 \mathrm{~m}$ to $1045 \mathrm{~m}$. W43-MM1 was imaged with a $78^{\prime \prime} \times 53^{\prime \prime}(2.1 \mathrm{pc} \times 1.4 \mathrm{pc}) \mathrm{mo}-$ saic composed of 33 fields with the $12 \mathrm{~m}$ array and 11 fields with ACA. In the $12 \mathrm{~m}$ configuration, the primary beam is of $26.7^{\prime \prime}$ (45.8" with ACA) and the maximum detectable scale is of $12^{\prime \prime}$ ( $21^{\prime \prime}$ with ACA). Parameters of the four spectral windows used in this study are presented in Table 1. Data were reduced in CASA 4.3.1, applying manual and self-calibration scripts.

We then used the CLEAN algorithm with a robust weight of 0.5 and the multiscale option to minimize interferometric artifacts due to missing short spacings. Core extraction was carried out on the $12 \mathrm{~m}$ continuum data providing the best resolution (synthesized beam of $0.37^{\prime \prime} \times 0.53^{\prime \prime}, \sim 2400$ au at $5.5 \mathrm{kpc}$ ) and the best sensitivity $\left(0.13 \mathrm{mJy}\right.$ beam $^{-1}$ on the $1.9 \mathrm{GHz}$ averaged map). For the molecular line studies we used the merged $(12 \mathrm{~m}+7 \mathrm{~m})$ data with characteristics given in Table 1 .

\section{Result and analysis}

\subsection{Core extraction and mass estimation}

Compact sources were extracted using getsources (v1.140127), a multiscale, multiwavelength source-extraction algorithm (Men'shchikov et al. 2012). The final getsources catalogs of the W43-MM1 cloud contains 131 reliable sources presented in Motte et al. (2018b). Among these sources, 13 high-mass cores with masses from 16 to $100 M_{\odot}$ are expected to form highmass stars, assuming a 50\% core-to-star efficiency for these very high-density cores. They have diameters ranging from 1200 to 2600 au once deconvolved from the $0.44^{\prime \prime}$ beam. These cores are outlined with ellipses on the $1.3 \mathrm{~mm}$ continuum map of Fig. 1 . We chose to focus on cores \#3 and \#6, two high-mass cores located at the western tip of the main filament (see Fig. 1), where the blending associated with clustering is minimum. Table 2 lists their coordinates, Gaussian full width at half maximum (FWHM) sizes, peak, and integrated fluxes.

The (gas + dust) mass of a core, having uniform opacity throughout its solid angle, is given by

$$
M_{\text {core }}=-\frac{\Omega_{\mathrm{b}} d^{2}}{\kappa_{1.3 \mathrm{~mm}}} \ln \left(1-\frac{S_{1.3 \mathrm{~mm}}^{\text {peak }}}{\Omega_{\mathrm{b}} B_{1.3 \mathrm{~mm}}\left(T_{\mathrm{dust}}\right)}\right) \times \frac{S_{1.3 \mathrm{~mm}}^{\text {int }}}{S_{1.3 \mathrm{~mm}}^{\text {peak }}},
$$

where $\Omega_{\mathrm{b}}$ is the solid angle of the beam, $d=5.5 \mathrm{kpc}$ is the distance to the core, $\kappa_{1.3 \mathrm{~mm}}$ is the dust opacity per unit of (gas + dust) mass at $1.3 \mathrm{~mm}, S_{1.3 \mathrm{~mm}}^{\text {int }}$ and $S_{1.3 \mathrm{~mm}}^{\text {peak }}$ are the peak and integrated fluxes of the core and $B_{1.3 \mathrm{~mm}}\left(T_{\text {dust }}\right)$ is the Planck function at dust temperature $T_{\text {dust }}$.

The $1.3 \mathrm{~mm}$ continuum flux of a core arises mainly from thermal dust emission, which is generally optically thin. This is no longer the case for the densest, most massive cores. Equation (1) thus includes a correction for dust opacity, which increases the mass of cores \#3 and \#6 by $20 \%$. Fluxes given in Table 2 are also corrected for contamination by free-free and line emission (see details in Motte et al. 2018b). The mean dust
Table 1. Parameters of the merged data spectral windows.

\begin{tabular}{|c|c|c|c|c|c|}
\hline \multirow{2}{*}{$\begin{array}{l}\text { Spectral } \\
\text { window }\end{array}$} & \multirow{2}{*}{$\begin{array}{c}v_{\mathrm{obs}} \\
(\mathrm{GHz})\end{array}$} & \multirow{2}{*}{$\begin{array}{c}\text { Bandwidth } \\
(\mathrm{MHz})\end{array}$} & \multicolumn{2}{|c|}{ Resolution } & \multirow{2}{*}{$\mathrm{rms}_{a}$} \\
\hline & & & $\left({ }^{\prime \prime}\right)$ & $\left(\mathrm{km} \mathrm{s}^{-1}\right)$ & \\
\hline $\mathrm{SiO}(5-4)$ & 217.033 & 234 & 0.48 & 0.3 & 2.5 \\
\hline $\mathrm{CO}(2-1)$ & 230.462 & 469 & 0.46 & 1.3 & 3.1 \\
\hline${ }^{13} \mathrm{CS}(5-4)$ & 231.144 & 469 & 0.46 & 0.3 & 3.1 \\
\hline Continuum & 233.4 & 1875 & 0.43 & 1.3 & 1.9 \\
\hline
\end{tabular}

Notes. ${ }^{(a)} 1 \sigma \mathrm{rms}$ in $\left(\mathrm{mJy}_{\text {beam }}{ }^{-1}\right) .1 \mathrm{mJy}$ beam $^{-1}$ corresponds to $0.12 \mathrm{~K}$ at $233.4 \mathrm{GHz}$.

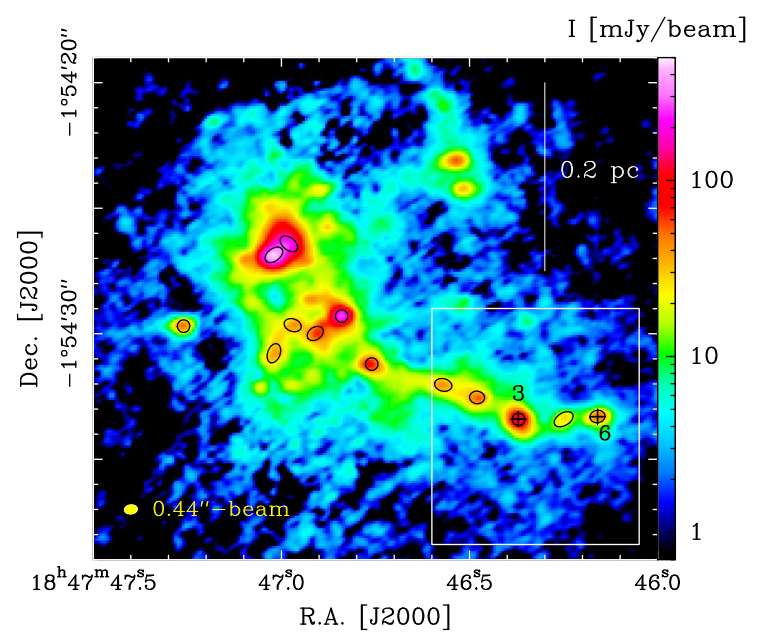

Fig. 1. Thirteen high-mass cores $\left(M_{\text {core }}>16 M_{\odot}\right.$, black ellipses $)$ discovered in the W43-MM1 protocluster (see Motte et al. 2018b). The white box outlines the zoom of Figs. 2a,b toward two very massive cores in the least clustered part of the main filament (\#3 and \#6, black crosses).

temperature of cores was estimated by applying the Bayesian PPMAP procedure (Marsh et al. 2015) to all existing continuum data of W43-MM1 (see Fig. 3 of Motte et al. 2018b). We adopted $\kappa_{1.3 \mathrm{~mm}}=0.01 \mathrm{~cm}^{2} \mathrm{~g}^{-1}$ as being the most appropriate for the high-density, $n_{\mathrm{H}_{2}} \sim 10^{9} \mathrm{~cm}^{-3}$, cool to warm, $20-40 \mathrm{~K}$, cores. The absolute uncertainties on core masses is estimated to be about a factor of two. Table 2 lists the assumed temperatures and derived masses, $M_{\text {core }}=59 M_{\odot}$ and $56 M_{\odot}$ for cores \#3 and \#6, respectively.

\subsection{Virial mass and gravitational boundedness}

To qualify as stellar progenitors, cores must be gravitationally bound. We characterize the isotropic turbulence of cores listed in Table 2 using the $J=5-4$ transition of ${ }^{13} \mathrm{CS}$, a good tracer of high-density gas (critical density $\sim 10^{6} \mathrm{~cm}^{-3}$ ). The ${ }^{13} \mathrm{CS}$ emission peaks toward high-mass cores but is also detected along their parental filament and their outflow. We thus subtracted, from each core spectrum, the contribution of the core parental filament and outflow before fitting a Gaussian (see Fig. A.1). The line FWHM given in Table 2 corresponds to the average of the fits before and after this subtraction. The line widths are large, $\Delta V_{{ }^{13} \mathrm{CS}} \sim 4.0 \mathrm{~km} \mathrm{~s}^{-1}$ and $\sim 3.3 \mathrm{~km} \mathrm{~s}^{-1}$, for cores \#3 and \#6, respectively.

The virial mass, $M_{\mathrm{vir}}$, is measured as

$M_{\mathrm{vir}}=3\left(\frac{5-2 n}{3-n}\right) \frac{\sigma_{\mathrm{NT}}^{2} F W H M}{G}$, 
Table 2. Cores' main characteristics derived from ALMA observations.

\begin{tabular}{|c|c|c|c|c|c|c|c|c|c|c|}
\hline Cores & $\begin{array}{c}\text { RA } \\
(\mathrm{J} 2000)\end{array}$ & $\begin{array}{c}\text { Dec } \\
(\mathrm{J} 2000)\end{array}$ & $\begin{array}{c}F W H M \\
(\mathrm{au})\end{array}$ & $\begin{array}{c}S_{1.3 \mathrm{~mm}}^{\text {peak }} \\
\left(\mathrm{mJy}^{-1} \text { beam }^{-1}\right)\end{array}$ & $\begin{array}{l}S_{1.3 \mathrm{~mm}}^{\mathrm{int}} \\
(\mathrm{mJy}) \\
\end{array}$ & $\begin{array}{c}T_{\text {dust }} \\
(\mathrm{K})\end{array}$ & $\begin{array}{l}M_{\text {core }} \\
\left(M_{\odot}\right)\end{array}$ & $\begin{array}{c}\Delta V_{13} \mathrm{CS} \\
\left(\mathrm{km} \mathrm{s}^{-1}\right)\end{array}$ & $\alpha_{\mathrm{vir}}{ }^{a}$ & $\begin{array}{l}\text { Outflow lobes } \\
(\mathrm{pc})\end{array}$ \\
\hline$\# 3$ & $18: 47: 46.37$ & $-1: 54: 33.41$ & 1200 & $109 \pm 2$ & $222 \pm 2$ & $45 \pm 2$ & $59 \pm 4$ & $4.0 \pm 0.3$ & 0.2 & 0.12 \\
\hline$\# 6$ & $18: 47: 46.16$ & $-1: 54: 33.30$ & 1300 & $46.8 \pm 0.8$ & $94 \pm 1$ & $23 \pm 2$ & $56 \pm 9$ & $3.3 \pm 0.2$ & $0.2-0.3$ & $<0.01$ \\
\hline
\end{tabular}

Notes. ${ }^{(a)}$ Virial parameter, $\alpha_{\text {vir }}=M_{\text {vir }} / M_{\text {core }}$, calculated with density indexes of $n=2$ for both cores and $n=0$ for core \#6.

where $n$ is the index of the density profile $\left(\rho \propto r^{-n}\right), \sigma_{\mathrm{NT}}$ is the 1D nonthermal velocity dispersion, FWHM is the deconvolved size of cores, and $G$ is the gravitational constant. We assume a density index of $n=2$ (case of a centrally concentrated protostar) for both cores and $n=0$ (case of a flat starless core) for core \#6, whose nature could be prestellar (see Sect. 4). The parameter $\sigma_{\mathrm{NT}}$ is derived by subtracting the thermal component from the total velocity dispersion, $\sigma_{\mathrm{NT}}^{2}=\sigma^{2}-\sigma_{\mathrm{th}}^{2}=\frac{\Delta V_{13} \mathrm{CS}}{8 \ln (2)}-\frac{k_{\mathrm{B}} T_{\mathrm{dust}}}{\mu_{13 \mathrm{CS}} m_{\mathrm{H}}}$, where $\mu_{13} \mathrm{CS}=45$ is the ${ }^{13} \mathrm{CS}$ molecular weight, $k_{\mathrm{B}}$ is the Boltzmann constant, and $m_{\mathrm{H}}$ is the hydrogen mass. Given that their virial parameters are well below unity, $\alpha_{\mathrm{vir}}=M_{\mathrm{vir}} / M_{\text {core }}=$ $0.2-0.3$, even with a factor of two uncertainty on masses, cores \#3 and \#6 are gravitationally bound and could be collapsing if no extra support, such as from magnetic fields, prevents it.

\subsection{Search for signposts of protostellar activity}

Gas outflows associated with accretion are certainly the best, systematic signpost of protostellar activity. We integrated the high-velocity line wings of $\mathrm{CO}(2-1)$ and $\mathrm{SiO}(5-4)$ around the systemic velocities $V_{\mathrm{LSR}} \simeq 96 \mathrm{~km} \mathrm{~s}^{-1}$ for core \#6 and $98 \mathrm{~km} \mathrm{~s}^{-1}$ for core \#3. The resulting lobes of bipolar outflows shown in Figs. 2a,b present an excellent agreement between the $\mathrm{CO}(2-1)$ and $\mathrm{SiO}(5-4)$ line tracers. While core \#3 unambiguously drives a well-developed outflow (see Figs. 2a,b and Table 2), the weak blue-shifted emission detected both in $\mathrm{CO}(2-1)$ and $\mathrm{SiO}(5-4)$ toward core \#6 is probably not associated with this core. As shown in Fig. A.2, the $\mathrm{SiO}(5-4)$ emission consists of two components. One decreasing from core \#18 to location A (see Fig. 2b) is associated with the outflow of core \#18. Another component peaking at location $\mathrm{B}$ could be associated with low-velocity shocks such as those observed by Louvet et al. (2016). Core \#6 thus seems to lack protostellar outflow but more sensitive and higher resolution observations (see Table 1) will be needed to confirm this finding.

The detection of line forests in spectral bands indicates molecular complexity (e.g., Herbst \& van Dishoeck 2009) and is often used to pinpoint hot cores. Figure 3 shows the $1.9 \mathrm{GHz}$ spectral band at $233.5 \mathrm{GHz}$ for cores \#3 and \#6. Very few lines are detected toward core \#6: few transition lines associated with methanol and methyl formate are detected at a $\sim 3 \sigma$ level in the continuum spectral window of core \#6. A careful analysis and modeling of the peculiar chemical complexity toward this core (Molet et al., in prep.) aims to interpret it in terms of protostellar heating, small-scale, or even large-scale shocks. These features are in strong contrast with the line forest observed for core \#3 (see Fig. 3). We also performed a quantitative measurement of the richness of the line forest by comparing the flux integrated over a line-free composite band of $65 \mathrm{MHz}$ with the flux integrated over the $1.9 \mathrm{GHz}$ full band, consisting of the sum of the line-free plus line-contamination fluxes. Line contamination, estimated by its the ratio to the line-free continuum flux, is $>13 \%$ for core \#3 and 0\% toward core \#6 (Motte et al. 2018b).
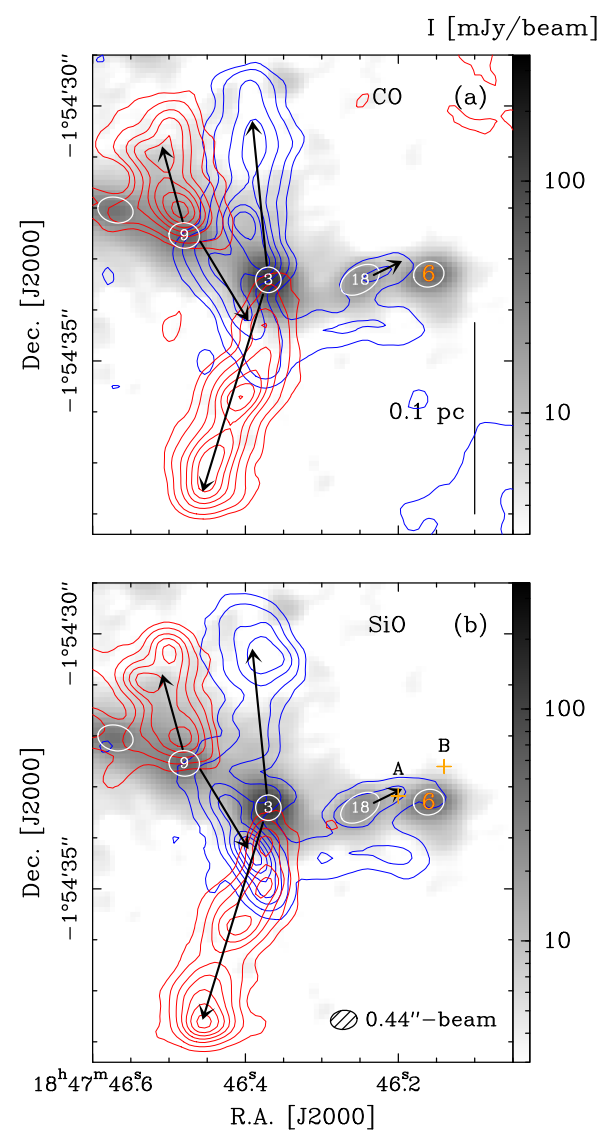

Fig. 2. Outflows driven by cores \#3, \#9, and \#18. Blue and red contours from the $\mathrm{CO}(2-1)$ (panel $a$ ) and $\mathrm{SiO}(5-4)$ (panel $b$ ) line wings are overplotted on the $1.3 \mathrm{~mm}$ continuum emission in grayscale. Lines are integrated over $38-88 \mathrm{~km} \mathrm{~s}^{-1}\left(60-90 \mathrm{~km} \mathrm{~s}^{-1}\right)$ for the CO (resp. $\mathrm{SiO})$ blue contours, and over $108-158 \mathrm{~km} \mathrm{~s}^{-1}\left(104-134 \mathrm{~km} \mathrm{~s}^{-1}\right)$ for the $\mathrm{CO}$ (resp. $\mathrm{SiO}$ ) red contours. $\mathrm{CO}$ contour levels are, in units of $\sigma_{\mathrm{CO}}=86 \mathrm{mJy}$ beam $^{-1} \mathrm{~km} \mathrm{~s}^{-1}, 6$ and $15-115$ by 20 steps. They are for $\mathrm{SiO}$, in unit of $\sigma_{\mathrm{SiO}}=18.5 \mathrm{mJy} \mathrm{beam}^{-1} \mathrm{~km} \mathrm{~s}^{-1}, 5$ and from 15 to 90 by 15 steps. Ellipses represent the FWHM diameter of cores extracted by getsources, crosses indicate locations $\mathrm{A}$ and $\mathrm{B}$, and arrows indicate the outflow directions.

These results suggest that core \#3 hosts a hot core while core \#6 may not yet.

\section{Discussion and conclusions}

The two cores discussed in this work have remarkably large masses, $\sim 60 M_{\odot}$, given their small diameters, $\sim 1300$ au (see Table 2), leading to extreme densities, $n_{\mathrm{H}_{2}} \sim 10^{9} \mathrm{~cm}^{-3}$. In Fig. 4 , cores \#3 and \#6 are compared to cores of a few $1000 \mathrm{au}$ sizes found within cold MDCs $(0.1 \mathrm{pc})$. These two cores are among the most massive detected so far at 1000-2000 au scales. They have high turbulent levels, $\sigma \sim 1.5 \mathrm{~km} \mathrm{~s}^{-1}$ corresponding 


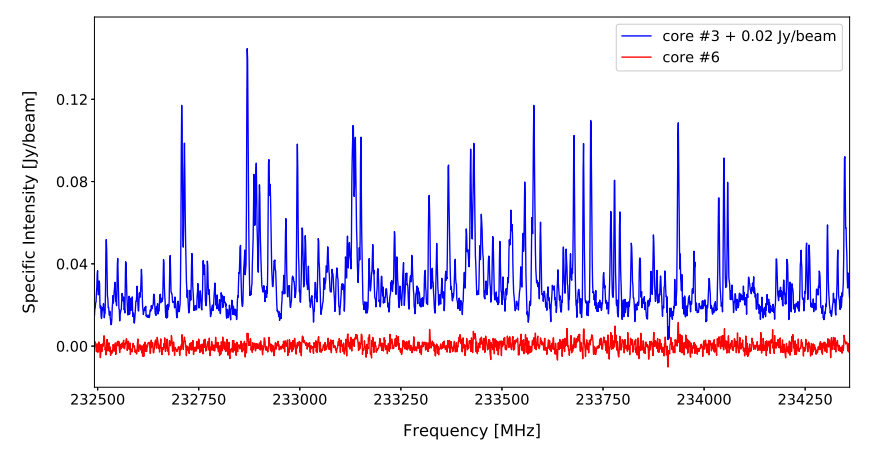

Fig. 3. Continuum-subtracted spectra of the $1.9 \mathrm{GHz}$ band used to estimate the $1.3 \mathrm{~mm}$ continuum emission of cores. Core \#3 stands out with a rich line forest, whereas very few lines are observed toward core \#6.

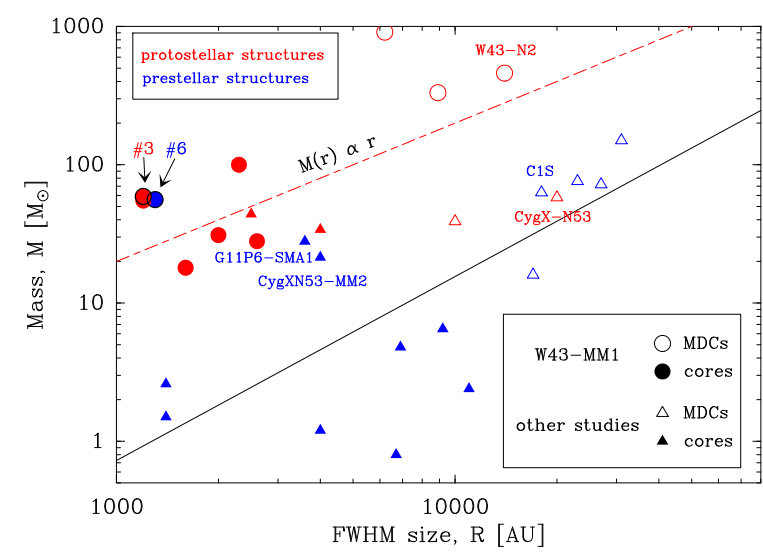

Fig. 4. Extremely massive $\sim 1000$ au-scale cores \#3 and \#6 and their parental MDCs in W43-MM1 (circles; Louvet et al. 2014; Motte et al. 2018b), compared to cores and MDCs of references studies (triangles; Bontemps et al. 2010; Tan et al. 2013; Wang et al. 2014; Tigé et al. 2017; Louvet et al. 2017). In these studies, masses are calculated from millimeter continuum with the same assumptions: dust temperature evaluated from large-scale spectral energy distribution fit (except for Wang et al. 2014 which use $\mathrm{NH}_{3}$ ), gas-to-dust ratio of 100 (except for Tan et al. 2013 which use 141), and an opacity index of 1.5. Cores \#3 and \#6 lie well above the empirical massive star formation threshold proposed by Kauffmann \& Pillai (2010; black line).

to Mach number $\sim 4.5$, which resemble those observed for ten-times larger young MDCs (e.g., Ragan et al. 2012; Kauffmann et al. 2013) and probably testifies to the high dynamics observed in W43-MM1 (e.g., Nguyen Luong et al. 2013; Louvet et al. 2016). Despite that, these cores have low virial parameters, $\alpha_{\mathrm{vir}}=0.2-0.3$, which proves they are gravitationally bound and could be collapsing if no extra support such as magnetic field prevents it. Core \#3 drives a bipolar outflow (see Figs. 2a,b) and displays molecular complexity possibly associated with hot core emission (see Fig. 3). In contrast, core \#6 shows no signs of protostellar activity. We currently cannot rule out the existence of an unresolved outflow and/or a small and embedded hot core, but core \#6 is, for now, an excellent highmass prestellar core candidate.

Core \#6 could therefore be among the very few examples representing the initial conditions of the McKee \& Tan (2003) model of high-mass star formation. In detail, this turbulent-core accretion model assumes that a $\sim 12000$ au core with $\sim 60 M_{\odot}$ and $\sigma \sim 1 \mathrm{~km} \mathrm{~s}^{-1}$ is quasi-statically assembled. Observationally, however, most of the $10000 \mathrm{au}$ cloud structures subfragment in protostars (Bontemps et al. 2010; Tan et al. 2016) or dissolve into low-mass cores (Kong et al. 2017; Louvet et al. 2017). In contrast core \#6, with its $56 M_{\odot}$ mass within $1300 \mathrm{au}$, keeps a large fraction, $\sim 12 \%$, of its parental MDC mass (W43N2, Louvet et al. 2014). This gas concentration tends to follow the $M(r) \propto r$ relation predicted for gravitationally bound cores such as Bonnor Ebert spheres (see Fig. 4). Core \#6 is also twice more massive than the few other high-mass prestellar core candidates, including CygXN53-MM2 (Bontemps et al. 2010; Duarte-Cabral et al. 2013) and G11P6-SMA1 (Wang et al. 2014).

Given that cores \#3 and \#6 have the same masses but different evolutionary stages, we use these cores to illustrate the evolutionary sequence proposed by Motte et al. (2018a). Core \#3 is a high-mass core, hosting a stellar embryo massive enough to power a hot core. This would then correspond to the IR-quiet or IR-bright protostellar core phases, which are steps 4 and 5 of this scenario. Core \#6 itself could either be a high-mass prestellar core or a very young high-mass protostellar core, hosting a very low-mass stellar embryo. While the existence of a highmass prestellar phase is refuted in Motte et al. (2018a), the second interpretation would put core \#6 in the IR-quiet protostellar core phase (step 4).

The mass-to-luminosity ratio, often used as an indicator of the evolutionary stage of cores, complies with core \#6 being younger than core \#3. Motte et al. (2018b) roughly evaluated the bolometric luminosity of cores from the total luminosity of W43-MM1, $\sim 2 \times 10^{4} L_{\odot}$ (Motte et al. 2003), divided between the cores in proportion to their line contamination. These luminosities, $L \sim 10 L_{\odot}$ for cores \#6 and $\sim 1000 L_{\odot}$ for cores \#3, lead to ratios of $M_{\text {core }} / L_{\text {bol }} \sim 6 M_{\odot} L_{\odot}^{-1}$ and $\sim 0.06 M_{\odot} L_{\odot}^{-1}$ for cores \#6 and \#3, respectively. These decreasing ratios, for cores of similar sizes and masses, indicates an increasing proportion of gas in the stellar embryo relative to the cold core and thus an evolution in time through the high-mass star-formation process.

We have proposed that core \#6 could represent the initial conditions of the turbulent-core monolithic-collapse model (McKee \& Tan 2003). However, since W43-MM1 is a very dynamical cloud (Louvet et al. 2016), its cores are expected to simultaneously collapse and grow in mass from their surrounding cloud gas. Therefore, core \#6 may be more consistent with the earliest stage of the accretion-stream model of Smith et al. (2009) and Vázquez-Semadeni et al. (2017) than with the quasistatic preassembled core taken for initial conditions in the model of McKee \& Tan (2003).

Acknowledgements. We thank A. Men'shchikov for his help on getsources This paper makes use of the following ALMA data: \#2013.1.01365.S. ALMA is a partnership of ESO (representing its member states), NSF (USA), and NINS (Japan), together with NRC (Canada), MOST and ASIAA (Taiwan), and KASI (Republic of Korea), in cooperation with the Republic of Chile. The Joint ALMA Observatory is operated by ESO, AUI/NRAO, and NAOJ. This project has received funding from the European Union's Horizon 2020 research and innovation program StarFormMapper under grant agreement No 687528. This work was supported by the Programme National de Physique Stellaire and Physique et Chimie du Milieu Interstellaire (PNPS and PCMI) of CNRS/INSU (with INC/INP/IN2P3) co-funded by CEA and CNES.

\section{References}

Bonnell, I. A., \& Bate, M. R. 2006, MNRAS, 370, 488 Bontemps, S., Motte, F., Csengeri, T., \& Schneider, N. 2010, A\&A, 524, A18 Csengeri, T., Bontemps, S., Schneider, N., Motte, F., \& Dib, S. 2011, A\&A, 527, A135

Duarte-Cabral, A., Bontemps, S., Motte, F., et al. 2013, A\&A, 558, A125 Henshaw, J. D., Caselli, P., Fontani, F., Jiménez-Serra, I., \& Tan, J. C. 2014, MNRAS, 440, 2860

Herbst, E., \& van Dishoeck, E. F. 2009, ARA\&A, 47, 427

Kauffmann, J., \& Pillai, T. 2010, ApJ, 723, L7 
T. Nony et al.: Detection of a high-mass prestellar core candidate in W43-MM1

Kauffmann, J., Pillai, T., \& Goldsmith, P. F. 2013, ApJ, 779, 185 Kong, S., Tan, J. C., Caselli, P., et al. 2017, ApJ, 834, 193

Louvet, F., Motte, F., Hennebelle, P., et al. 2014, A\&A, 570, A15 Louvet, F., Motte, F., Gusdorf, A., et al. 2016, A\&A, 595, A122

Louvet, F., Neupane, S., Motte, F., et al. 2017, A\&A, submitted Marsh, K., Whitworth, A., \& Lomax, O. 2015, MNRAS, 454, 4282 McKee, C. F., \& Tan, J. C. 2003, ApJ, 585, 850

Men'shchikov, A., André, P., Didelon, P., et al. 2012, A\&A, 542, A81

Motte, F., Schilke, P., \& Lis, D. C. 2003, ApJ, 582, 277

Motte, F., Bontemps, S., \& Louvet, F. 2018a, ARA\&A, 56, 41

Motte, F., Nony, T., Louvet, F., et al. 2018b, Nat. Astron., 2, 478

Nguyen Luong, Q., Motte, F., Schuller, F., et al. 2011, A\&A, 529, A41
Nguyen Luong, Q., Motte, F., Carlhoff, P., et al. 2013, ApJ, 775, 88 Peretto, N., Fuller, G. A., Duarte-Cabral, A., et al. 2013, A\&A, 555, A112 Ragan, S. E., Heitsch, F., Bergin, E. A., \& Wilner, D. 2012, ApJ, 746, 174 Schneider, N., Csengeri, T., Bontemps, S., et al. 2010, A\&A, 520, A49 Smith, R. J., Longmore, S., \& Bonnell, I. 2009, MNRAS, 400, 1775

Tan, J. C., Kong, S., Butler, M. J., Caselli, P., \& Fontani, F. 2013, ApJ, 779, 96 Tan, J. C., Kong, S., Zhang, Y., et al. 2016, ApJ, 821, L3

Tigé, J., Motte, F., Russeil, D., et al. 2017, A\&A, 602, A77

Vázquez-Semadeni, E., González-Samaniego, A., \& Colín, P. 2017, MNRAS, 467,1313

Wang, K., Zhang, Q., Testi, L., et al. 2014, MNRAS, 439, 3275

Zhang, B., Moscadelli, L., Sato, M., et al. 2014, ApJ, 781, 89 


\section{Appendix A}
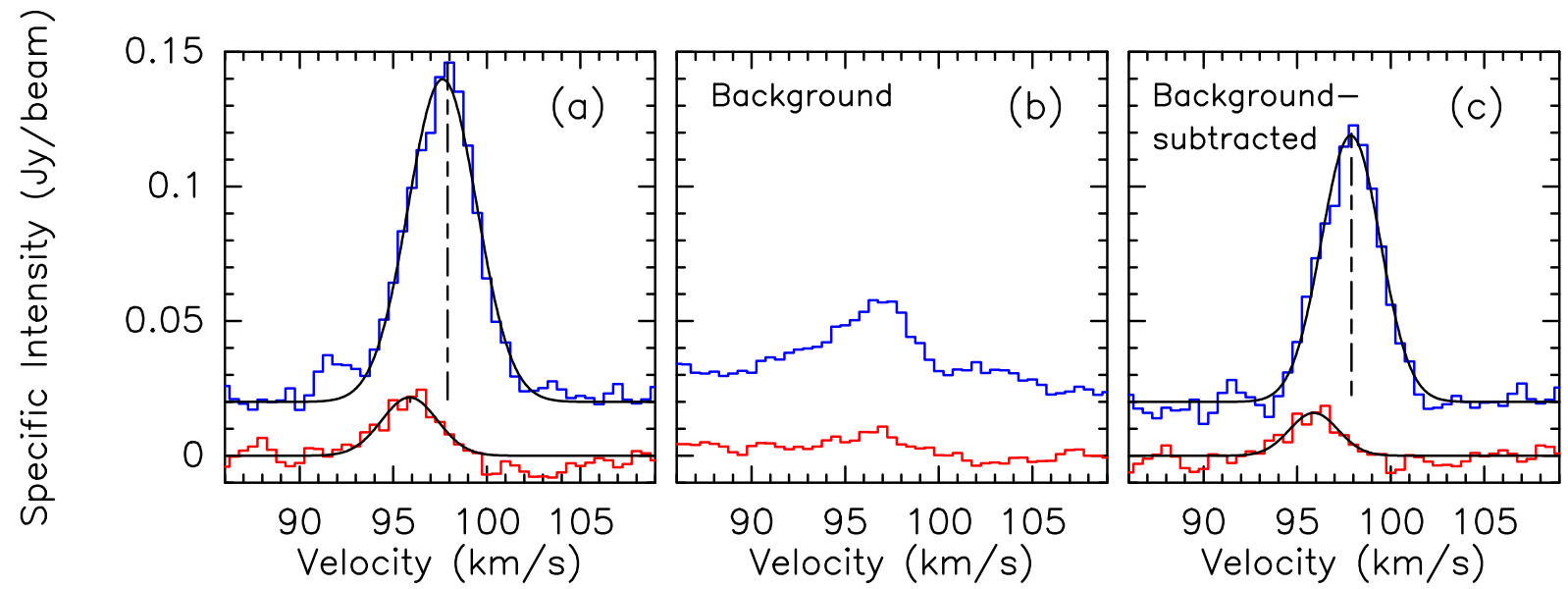

Fig. A.1. Spectra of the ${ }^{13} \mathrm{CS}$ line detected toward the continuum peak of core \#3 (in blue with a $0.02 \mathrm{Jy}_{\text {beam }}{ }^{-1}$ offset) and core \#6 (in red), before (panel a) and after (panel c) subtraction of their parental filament and outflow emission (panel b). Black curves are the Gaussian fits used to measure the line widths of the cores: $\Delta V_{{ }^{13} \mathrm{CS}}=4.3 \mathrm{~km} \mathrm{~s}^{-1}$ and $3.7 \mathrm{~km} \mathrm{~s}^{-1}$ for core $\# 3, \Delta V_{{ }^{13} \mathrm{CS}}=3.5 \mathrm{~km} \mathrm{~s}^{-1}$ and $3.1 \mathrm{~km} \mathrm{~s}^{-1}$ for core \#6 before and after background subtraction, respectively.

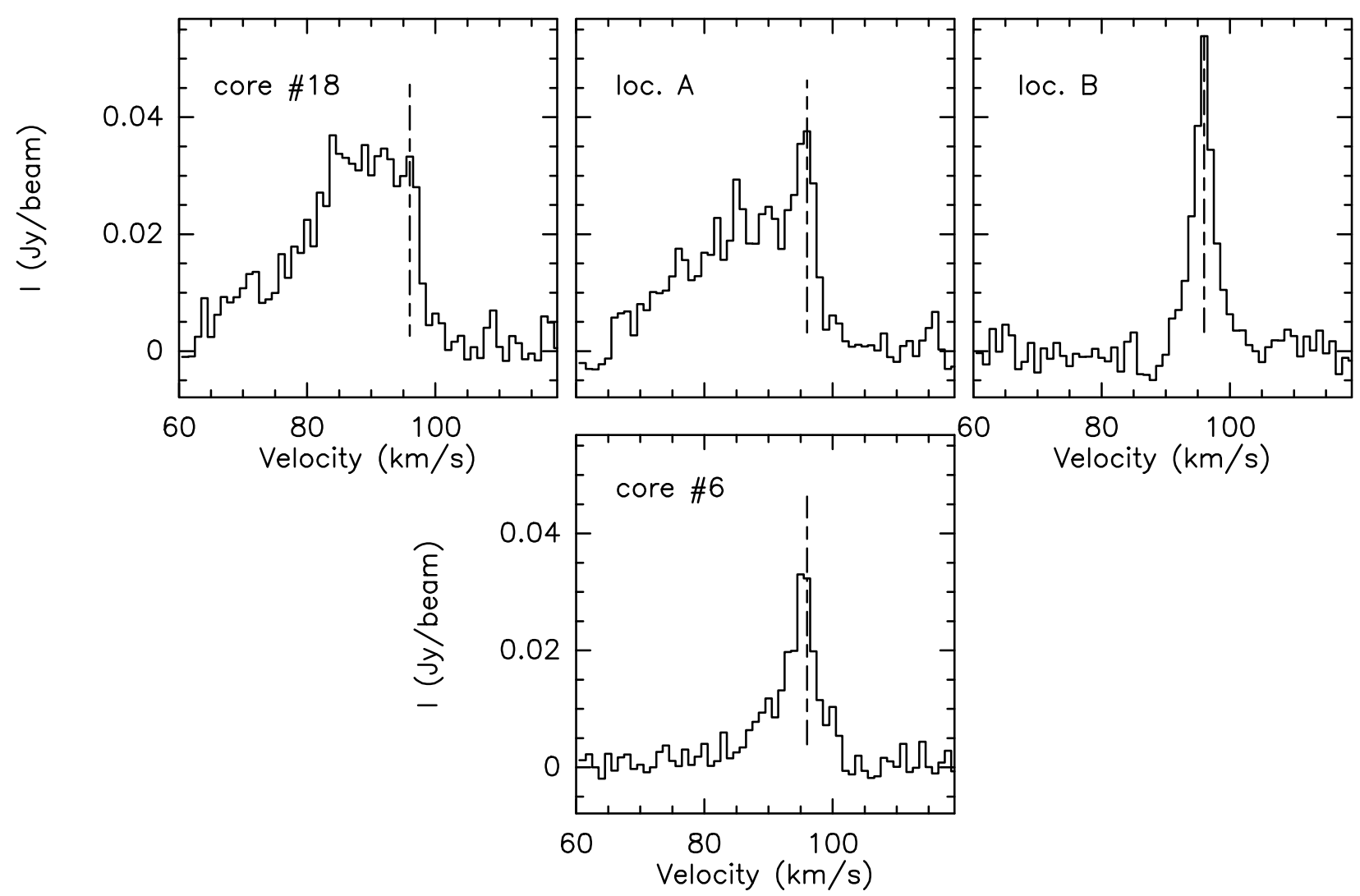

Fig. A.2. Spectra of the $\mathrm{SiO}(5-4)$ line toward the continuum peak of cores \#6 and \#18 and toward locations A and B (see Fig. 2b and Sect. 3.3). The vertical dashed lines indicate the velocity of the $\mathrm{SiO}$ line at location $\mathrm{B}\left(96 \mathrm{~km} \mathrm{~s}^{-1}\right)$. 